\title{
The Dynamic Evaluation of Tilted Support Spring Nonlinear System with Critical Components under the Action of a Rectangular Pulse
}

\author{
Ningning Duan, ${ }^{1}$ Shuang Song, ${ }^{1}$ and Anjun Chen ${ }^{1,2}$ \\ ${ }^{1}$ School of Mechanical Engineering, Jiangnan University, Wuxi, Jiangsu 214122, China \\ ${ }^{2}$ China National Control and Test Center for Packaging Quality, Wuxi, Jiangsu 214122, China \\ Correspondence should be addressed to Anjun Chen; caj62@163.com
}

Received 7 November 2013; Revised 26 February 2014; Accepted 26 February 2014; Published 25 March 2014

Academic Editor: Weichao Sun

Copyright (C) 2014 Ningning Duan et al. This is an open access article distributed under the Creative Commons Attribution License, which permits unrestricted use, distribution, and reproduction in any medium, provided the original work is properly cited.

Dimensionless nonlinear dynamical equations of a tilted support spring nonlinear system with critical components were obtained under the action of a rectangular pulse, and the numerical results of the shock response were studied using Runge-Kutta method. To evaluate the dynamic characteristics of critical components, a new concept of three-dimensional shock response spectra was proposed, where the ratio of the maximum shock response acceleration of critical components to the peak pulse acceleration, the pulse duration, and the frequency ratio were three basic parameters of three-dimensional shock response spectra. Based on the numerical results, the effects of the angle, the peak pulse acceleration, the mass ratio, the frequency ratio, and the pulse duration on the shock response spectra were discussed.

\section{Introduction}

A tilted support spring nonlinear system has been widely applied in the vibration reduction of vehicle suspension and is an improved cushion structure, which is used to connect an inner body with an outer body by four tilted support springs. The system usually shows superior absorber effects to a linear system and is applied to protect precise instruments from damage since the 1960s. In the automotive engineering, the absorber system of an engine generally adopts the angle $70^{\circ}-90^{\circ}$ of the tilted support spring nonlinear system, which provides effective absorber protections for the engine. According to extensive researches, a product is damaged in the transport, mainly because of shock and vibration. So, to estimate dynamic characteristics of the system, the concept of a shock response spectrum is proposed and used by many specialists. Alexander [1] presented that the shock response spectrum was used to analyze the frequency response of an acceleration-time-history record to probe into the maximum dynamic response. Tuma at al. [2] proposed the calculation method of the shock response spectra, which is used to deal with employing Signal Analyzer. Xu et al. [3] analyzed the high pressure regimes of porous material in shock wave response by using a microscopic method. To evaluate the influence of the impulse and geometric parameters on shock characteristics of plates under impulse loads, the model and shock response spectra of the system were carried on by Botta and Cerri [4]. Goetz and Matous [5] presented that the model of two-layered cellular materials under impulse loads was conducted to give insights into the shock characteristics of the system and to optimize the system. A dynamic model of the corrugated paperboard cushioning packaging system was established by Wang et al. [6], and a comparison between the approximate solution of nonlinear equations and the numerical simulation results was made. Wang et al. [7] obtained a model for critical components of a nonlinear packaging system and then researched the effect of the mass ratio, the frequency ratio, and the damping ratio on threedimensional shock spectrum. As can be seen from the above analysis, most studies mainly focus on material nonlinearity at present, but the geometric nonlinearity caused by the structure of the system has barely been reported.

Shock and vibration are main factors causing damages of products in transport. It is extremely important to protect 


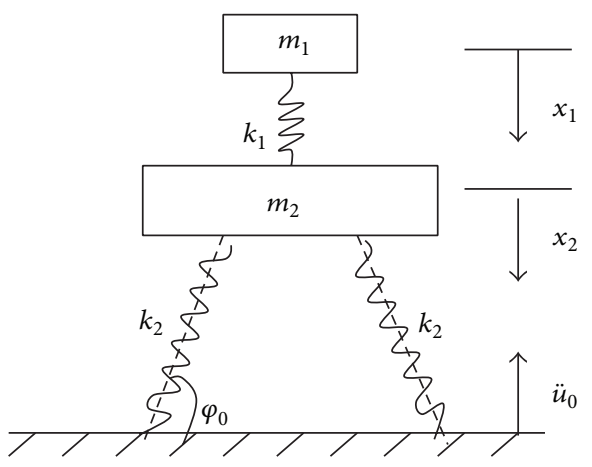

FIGURE 1: The model of the tilted support spring nonlinear system with critical components.

products from being destroyed by dynamic evaluation of a nonlinear system. Wang and $\mathrm{Hu}[8,9]$ obtained the shock response spectra and damage boundary curve in order to investigate the shock characteristics of various nonlinear materials under the action of typical pulses. Wang and Chen [10] gained the model of suspension packaging system under rectangular pulse and further discussed the effects of the angle and the damping ratio on the shock response spectra and the acceleration response of dimensionless peak pulse.

However, the shock response spectrum of the tilted support spring system with critical components has hardly been discussed until today. In this paper, the dimensionless nonlinear dynamical equations for the tilted support spring system with critical components are obtained under the action of a rectangular pulse. According to the evaluation methodology [8-11], a new concept of three-dimensional shock response spectra is gained and the effects of the peak pulse acceleration, the pulse duration, the angle, the mass ratio, and the frequency ratio on dynamic characteristics of critical components are discussed. The results provide theoretical foundations for the design of the tilted support spring nonlinear system.

\section{Modeling and Equations}

The tilted support spring nonlinear system with critical components is shown in Figure 1, where $m_{1}$ and $m_{2}$ denote the mass of critical components and the main body, respectively; $k_{1}$ is the linear elastic coefficient between critical components and the main body; $k_{2}$ is the linear elastic coefficient of the tilted support spring system; $l_{0}$ and $\varphi_{0}$ are the length and the support angle of the four tilted support springs before they are compressed.

Because the model of the system is principally affected by the vertical direction, the study on other directions of the system can be omitted, just like Figure 1. To facilitate the numerical analysis, the coordinate system is obtained, the static equilibrium position is treated as the original points, and the downward direction is regarded as the positive direction. Then, the vertical natural vibration dynamic equations [12] can be obtained as

$$
\begin{gathered}
m_{1} \frac{\mathrm{d}^{2} x_{1}}{\mathrm{~d} t^{2}}-k_{1}\left(x_{2}-x_{1}\right)=0 \\
m_{2} \frac{\mathrm{d}^{2} x_{2}}{\mathrm{~d} t^{2}}+2 k_{2}\left(a_{0} x_{2}+\frac{b_{0}}{l_{0}} x_{2}^{2}+\frac{c_{0}}{l_{0}^{2}} x_{2}^{3}\right)+k_{1}\left(x_{2}-x_{1}\right)=0
\end{gathered}
$$

where $a_{0}=\sin ^{2} \varphi_{0}, b_{0}=-3 / 2 \sin \varphi_{0} \cos ^{2} \varphi_{0}$, and $c_{0}=1 / 2(1-$ $\left.6 \sin ^{2} \varphi_{0}+5 \sin ^{4} \varphi_{0}\right)$, which is related to the angle of the system, namely, geometric structure nonlinearity.

To evaluate the dynamic characteristics of the system under the action of a rectangular pulse, the equations of the pulse is expressed as

$$
\ddot{u}_{0}= \begin{cases}\ddot{u}_{0 m} & 0 \leq t \leq t_{0} \\ 0 & t>t_{0}\end{cases}
$$

where $t_{0}$ is the pulse duration. Substitute (2) into (1); then the shock dynamic equations of the system can be derived as

$$
\begin{gathered}
m_{1} \frac{\mathrm{d}^{2} x_{1}}{\mathrm{~d} t^{2}}-k_{1}\left(x_{2}-x_{1}\right)=0 \\
m_{2} \frac{\mathrm{d}^{2} x_{2}}{\mathrm{~d} t^{2}} \\
+2 k_{2}\left[a_{0}\left(x_{2}+u_{0}\right)+\frac{b_{0}}{l_{0}}\left(x_{2}+u_{0}\right)^{2}+\frac{c_{0}}{l_{0}^{2}}\left(x_{2}+u_{0}\right)^{3}\right] \\
+k_{1}\left(x_{2}-x_{1}\right)=0,
\end{gathered}
$$

where $x_{1,2}(0)=0$ and $\mathrm{d} x_{1,2}(0) / \mathrm{d} t=0$ are initial conditions of the displacement and the velocity, respectively.

Letting $y_{1}=\left(x_{2}-x_{1}\right) / l_{0}, y_{2}=\left(x_{2}+u_{0}\right) / l_{0}, \omega_{1}=\sqrt{k_{1} / m_{1}}$, and $\omega_{2}=\sqrt{2 k_{2} / m_{2}}$ and combing with (3), the dimensionless nonlinear shock dynamic equations of the system under the action of a rectangular pulse can be shown as

$$
\begin{gathered}
\frac{\mathrm{d}^{2} y_{1}}{\mathrm{~d} \tau^{2}}+\left(a_{0} y_{2}+b_{0} y_{2}^{2}+c_{0} y_{2}^{3}\right)+\lambda_{1}^{2} \lambda_{2} y_{1}+\lambda_{1}^{2} y_{1}=0 \\
\frac{\mathrm{d}^{2} y_{2}}{\mathrm{~d} \tau^{2}}+\left(a_{0} y_{2}+b_{0} y_{2}^{2}+c_{0} y_{2}^{3}\right)+\lambda_{1}^{2} \lambda_{2} y_{1}=\beta \ddot{u}_{0},
\end{gathered}
$$

where $T=1 / \omega_{2}$ is taken as the period parameter; $\tau=$ $t / T$ is the dimensionless time parameter; $\tau_{0}=t_{0} / T$ is the dimensionless pulse duration parameter; $\lambda_{1}=\omega_{1} / \omega_{2}$ is the frequency ratio of the system; $\lambda_{2}=m_{1} / m_{2}$ is the mass ratio of the system; $\beta=T^{2} / l_{0}$ is defined as a characteristic parameter of the system; $\beta \ddot{u}_{0 m}$ is the dimensionless peak pulse acceleration, respectively.

According to (4), initial conditions of the dimensionless displacement and velocity can be transformed as $y_{1,2}(0)=0$ and $\mathrm{d} y_{1,2}(0) / \mathrm{d} \tau=0$, respectively. 


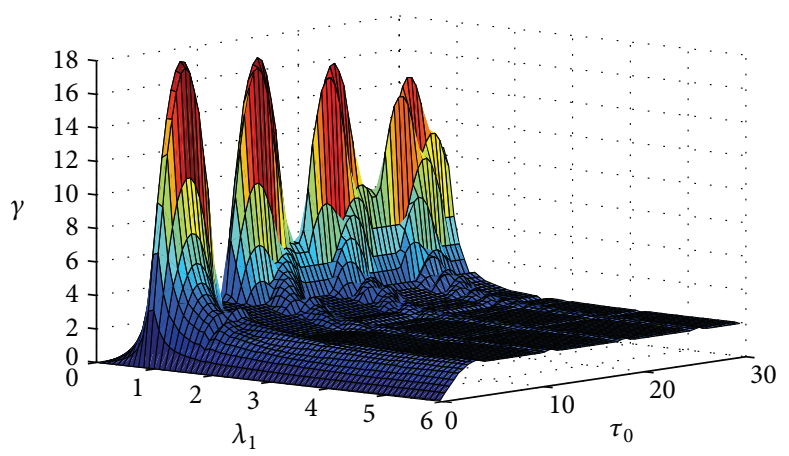

(a)

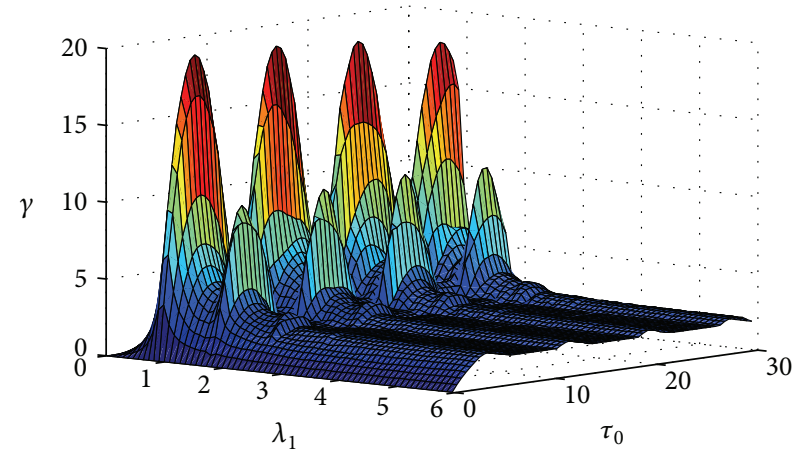

(c)

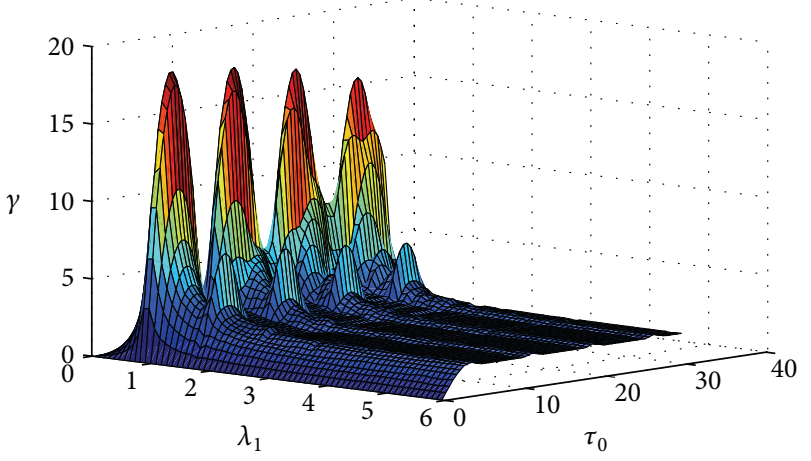

(b)

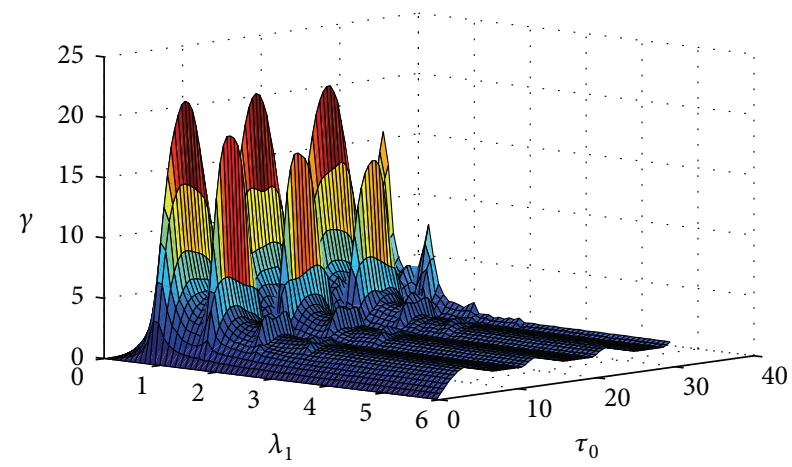

(d)

FIGURE 2: The effect of the peak pulse acceleration on three-dimensional shock response spectra of criminal components when (a) $\beta \ddot{u}_{0 m}=$ 0.01 , (b) $\beta \ddot{u}_{0 m}=0.05$, (c) $\beta \ddot{u}_{0 m}=0.1$, and (d) $\beta \ddot{u}_{0 m}=0.15 . \gamma$ : the ratio of the maximum shock response acceleration of critical components to the peak pulse acceleration, $\lambda_{1}$ : the frequency ratio of the system, and $\tau_{0}$ : the dimensionless pulse duration.

The expression of the dimensionless rectangular pulse is then in (4):

$$
\beta \ddot{u}_{0}= \begin{cases}\beta \ddot{u}_{0 m} & 0 \leq \tau \leq \tau_{0} \\ 0 & \tau>\tau_{0}\end{cases}
$$

\section{Dynamic Evaluation}

Based on the analysis of (4), it demonstrates that the dimensionless acceleration response of a product and its critical components is relevant to the angle of the system, the dimensionless peak pulse acceleration, the dimensionless pulse duration, the mass ratio, and the frequency ratio of the system. This section will chiefly concentrate on threedimensional shock response spectra of critical components, which can clearly reflect the dynamic characteristics of the tilted support spring nonlinear system caused by the geometry nonlinearity of the structure.

To evaluate the dynamic characteristics of the system under the action of a rectangular pulse, a new concept of three-dimensional shock response spectra is proposed, where the ratio of the maximum shock response acceleration of critical components to the peak pulse acceleration $\gamma=$ $\left(\ddot{x}_{1}\right)_{m} / \ddot{u}_{0 m}$ is regarded as the response index of the system, namely, dynamic amplification coefficient. Integrating the dimensionless parameters of the system $y_{1}=\left(x_{2}-x_{1}\right) / l_{0}$ and $y_{2}=\left(x_{2}+u_{0}\right) / l_{0}$ with (4), the dynamic amplification coefficient can be written as

$$
\begin{aligned}
\gamma & =\frac{\left(\mathrm{d}^{2} x_{1} / \mathrm{d} t^{2}\right)_{m}}{\mathrm{~d}^{2} u_{0 m} / \mathrm{d} t^{2}} \\
& =\frac{\left(\left(\mathrm{d}^{2} y_{2} / \mathrm{d} \tau^{2}\right)-\left(\mathrm{d}^{2} y_{1} / \mathrm{d} \tau^{2}\right)-\left(\beta\left(\mathrm{d}^{2} u_{0} / \mathrm{d} \tau^{2}\right)\right)\right)_{m}}{\beta\left(\mathrm{d}^{2} u_{0 m} / \mathrm{d} \tau^{2}\right)} \\
& =\frac{\left(\lambda_{1}^{2} y_{1}\right)_{m}}{\beta \ddot{u}_{0 m}} .
\end{aligned}
$$

The dimensionless pulse duration $\tau_{0}$ and the frequency ratio of the system $\lambda_{1}$ are considered to be two basic parameters of the three-dimensional shock response spectra. Based on the numerical results, the effects of the angle, the mass ratio, the frequency ratio, the dimensionless peak pulse acceleration, and the dimensionless pulse duration on shock response characteristics of critical components are discussed.

The dimensionless shock dynamic equations (4) are solved using fourth-order Runge-Kutta method. At the same time, set the dimensionless pulse duration $0 \leq \tau_{0} \leq 40$ and the frequency ratio of the system $0 \leq \lambda_{1} \leq 6$. When we choose the mass ratio $\lambda_{2}=0.01$ and the angle of the system $\varphi_{0}=$ $60^{\circ}$, the effect of the dimensionless peak pulse acceleration on the three-dimensional shock response spectra of critical components is given in Figure 2. 


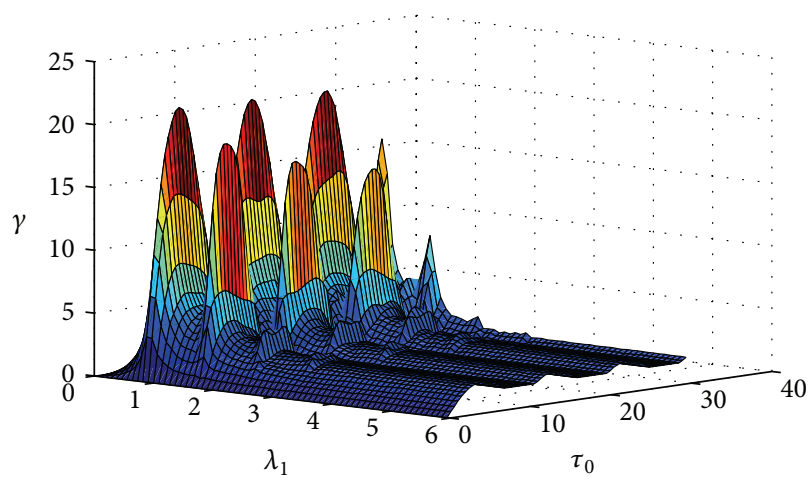

(a)

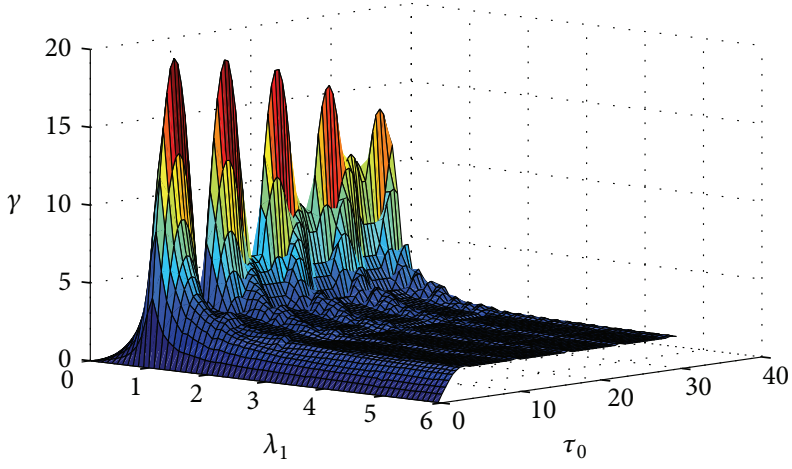

(c)

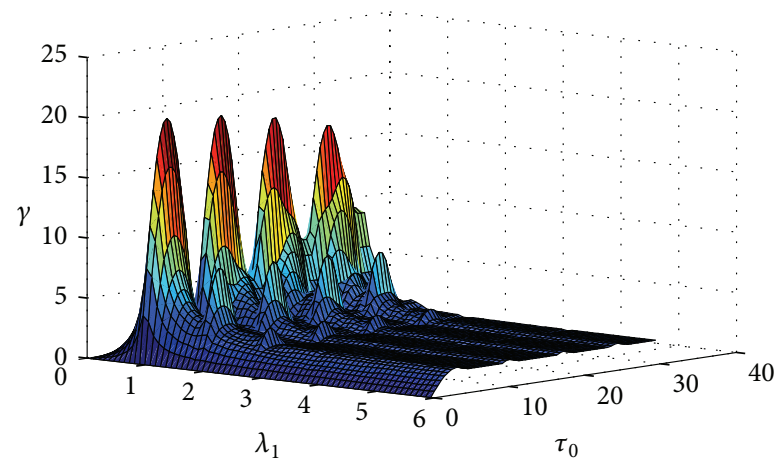

(b)

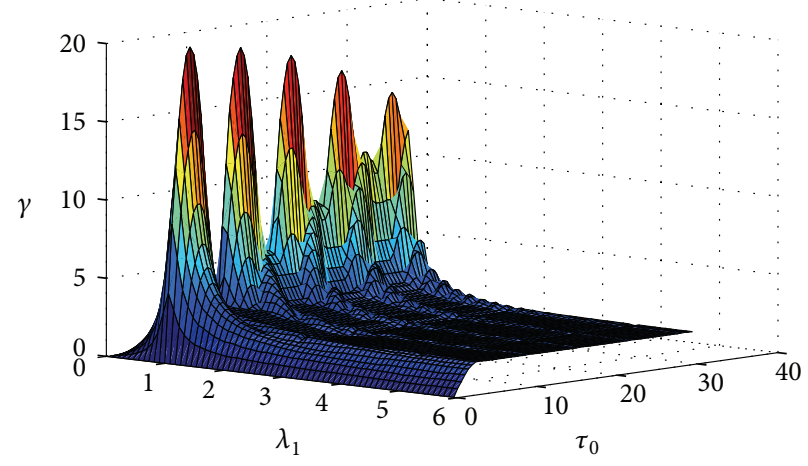

(d)

FIGURE 3: The effect of the angle of the tilted support spring system on three-dimensional shock response spectra of criminal components when (a) $\varphi_{0}=60^{\circ}$, (b) $\varphi_{0}=70^{\circ}$, (c) $\varphi_{0}=85^{\circ}$, and (d) $\varphi_{0}=90^{\circ} . \gamma$ : the ratio of the maximum shock response acceleration of critical components to the peak pulse acceleration, $\lambda_{1}$ : the frequency ratio of the system, and $\tau_{0}$ : the dimensionless pulse duration.

As is shown in Figure 2, when it is low frequency ratio, under the action of a rectangular pulse, increasing the dimensionless peak pulse acceleration can lead to noticeable fluctuation of the maximum shock response acceleration of critical components and arising more peaks of shock response spectra.

According to the previous researches, the discussions about the tilted support spring nonlinear system use the range of $60^{\circ}-90^{\circ}$. Therefore, under the conditions of the mass ratio $\lambda_{2}=0.01$ and the dimensionless peak pulse $\beta \ddot{u}_{0 m}=0.15$, the effect of the angle $\left(60^{\circ}, 70^{\circ}, 85^{\circ}, 90^{\circ}\right)$ on three-dimensional shock response spectra of critical components is revealed in Figure 3.

As is shown in Figure 3, comparing with linear system $\left(\varphi_{0}=90^{\circ}\right)$, decreasing the angle can remarkably aggravate the fluctuation of maximum shock response acceleration of critical components at low frequency ratio. Wu et al. [12] have researched the fact that the damping effect of the tilted support nonlinear spring is superior to the linear system, while the shock resistance function of the system decreases.

Figure 4 presents the effect of the mass ratio on threedimensional shock response spectra of critical components, setting the angle of the system $\varphi_{0}=60^{\circ}$ and the dimensionless peak pulse $\beta \ddot{u}_{0 m}=0.05$.

According to analysis of numerical results in Figure 4, it reveals that increasing the mass ratio can effectively restrain the maximum shock response acceleration of critical components at low frequency ratio.

Figures 2-4 show the effect of frequency ratio on threedimensional shock response spectra. In terms of tilted support spring nonlinear system with critical components, the maximum shock response acceleration of critical components under the action of a rectangular pulse is sensitive to low frequency ratio. That is to say, the three-dimensional shock response spectra of criminal components exist in sensitive areas.

\section{Conclusions}

By analyzing the three-dimensional shock response spectra of the tilted support spring nonlinear system with critical components, Figures 2-3 demonstrate that increasing the peak pulse acceleration or decreasing the angle can rapidly increase the maximum shock response acceleration of critical components, and the effect of low frequency ratio on shock response of the system is particularly sensitive. It shows in Figures 2-4 that increasing the frequency ratio of the system can obviously decrease the maximum shock response acceleration of critical components, and the peak of the shock response for critical components can be evidently decreased at the low frequency ratio by increasing the mass ratio of the system. In addition, Figures 2-4 can clearly show that, 


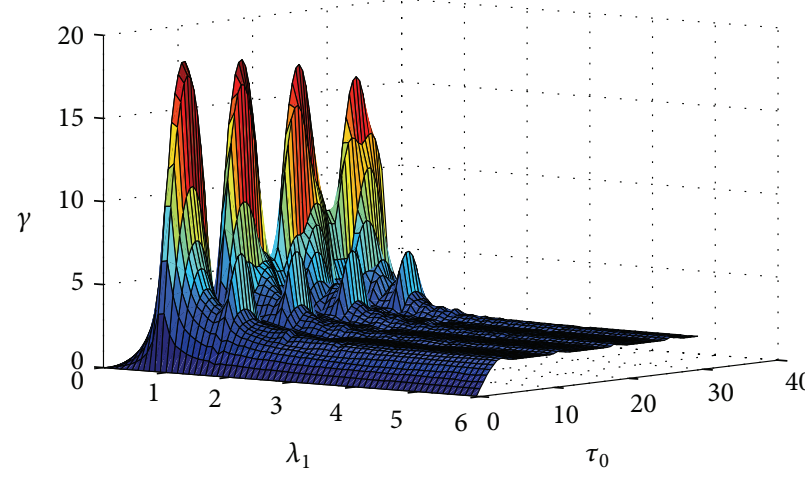

(a)

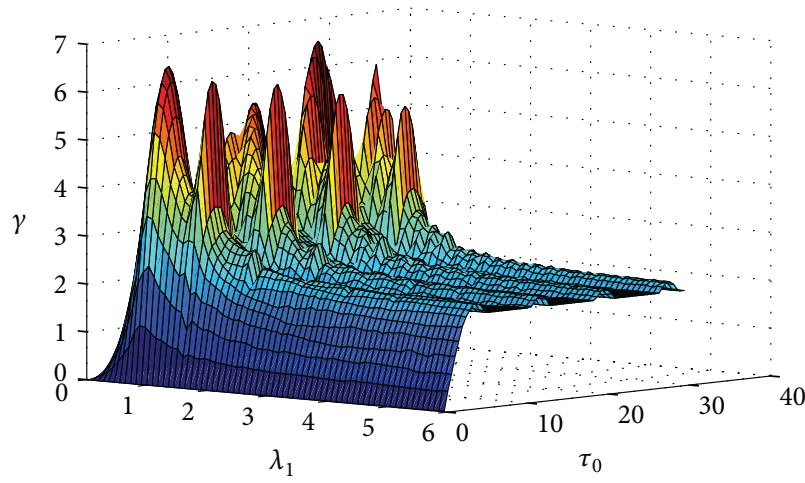

(c)

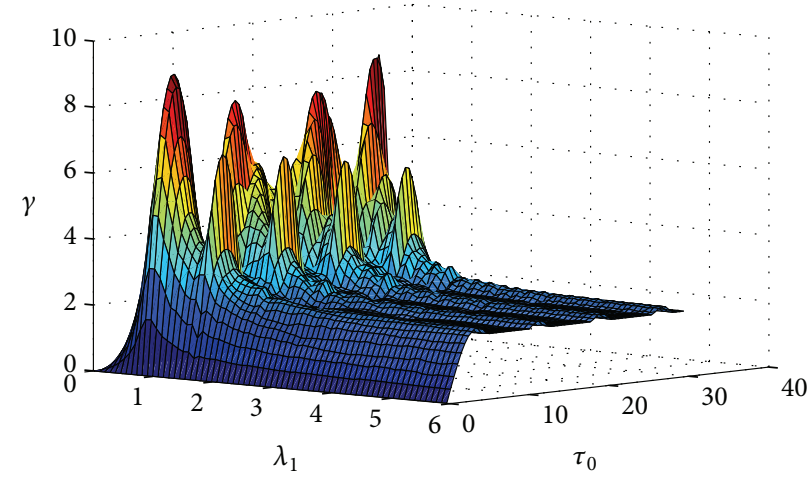

(b)

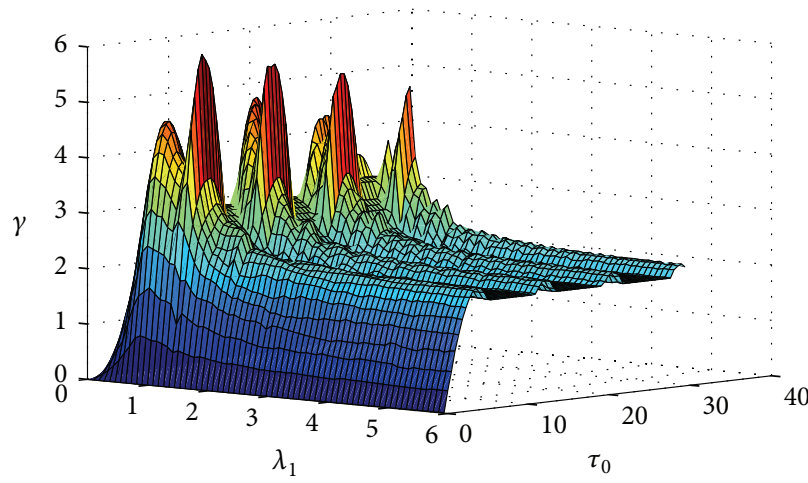

(d)

FIGURE 4: The effect of the mass ratio on three-dimensional shock response spectra of criminal components when (a) $\lambda_{2}=0.01$, (b) $\lambda_{2}=0.05$, (c) $\lambda_{2}=0.1$, and (d) $\lambda_{2}=0.2 . \gamma$ : the ratio of the maximum shock response acceleration of critical components to the peak pulse acceleration, $\lambda_{1}$ : the frequency ratio of the system, and $\tau_{0}$ : the dimensionless pulse duration.

with the increase of the dimensionless pulse duration, at low frequency ratio $\left(\lambda_{1}<5\right)$ the shock response acceleration of critical components can arise multiple peaks, while at high frequency ratio the shock response acceleration of critical components tends to be stable $(\gamma=2)$. Therefore, it is extremely necessary to control the above parameters adequately for decreasing the maximum shock response acceleration of critical components.

On the basis of the numerical analysis, the frequency ratio of the system is an important parameter. It is particularly significant for advancing shock resistance characteristics of critical components to control the frequency ratio suitably and increase the frequency ratio of the system as possible in the permissive condition (it is suggested that frequency ratio is $\lambda_{1}>5$ ).

\section{Conflict of Interests}

The authors declare that there is no conflict of interests regarding the publication of this paper.

\section{References}

[1] J. E. Alexander, "Shock response spectrum-a primer," Sound and Vibration, vol. 43, no. 6, pp. 6-15, 2009.
[2] J. Tuma, M. Babiuch, and P. Koci, "Calculation of a shock response spectra," Acta Montanistica Slovaca, vol. 16, no. 1, pp. 66-73, 2011.

[3] A. G. Xu, G. C. Zhang, H. Li, Y. Ying, and J. Zhu, "Dynamical similarity in shock wave response of porous material: from the view of pressure," Computers and Mathematics with Applications, vol. 61, no. 12, pp. 3618-3627, 2011.

[4] F. Botta and G. Cerri, "Shock response spectrum in plates under impulse loads," Journal of Sound and Vibration, vol. 308, no. 3-5, pp. 563-578, 2007.

[5] J. C. Goetz and K. Matous, "Shock analysis and optimization of two-layered cellular materials subject to pulse loading," International Journal of Impact Engineering, vol. 57, pp. 55-69, 2013.

[6] J. Wang, Z. W. Wang, F. Duan et al., "Dropping shock response of corrugated paperboard cushioning packaging system," Journal of Vibration and Control, vol. 19, no. 3, pp. 336-340, 2013.

[7] J. Wang, Z. W. Wang, L. X. Lu, Y. Zhu, and Y. G. Wang, "Three-dimensional shock spectrum of critical component for nonlinear packaging system," Shock and Vibration, vol. 18, no. 3, pp. 437-445, 2011.

[8] Z. W. Wang and C. Y. Hu, "Shock spectra and damage boundary curves for nonlinear package cushioning system," Packaging Technology and Science, vol. 12, no. 5, pp. 207-217, 1999.

[9] Z. W. Wang, "Shock spectra and damage boundary curves for hyperbolic tangent cushioning system and their important 
features," Packaging Technology and Science, vol. 14, no. 4, pp. 149-157, 2001.

[10] L. Wang and A. J. Chen, "The shock response spectra of the suspension packaging system under rectangular pulse," Journal of Applied Packaging Research, vol. 5, no. 4, pp. 237-246, 2012.

[11] Z. W. Wang and J. H. Jiang, "Evaluation of product dropping damage based on key component," Packaging Technology and Science, vol. 23, no. 4, pp. 227-238, 2010.

[12] X. Wu, Y. X. Luo, and Y. Wu, "Study on vertical nonlinear natural vibration of shock absorber system with tilted support spring," Journal of Vibration and Shock, vol. 27, no. 8, pp. 85-87, 2008 (Chinese). 


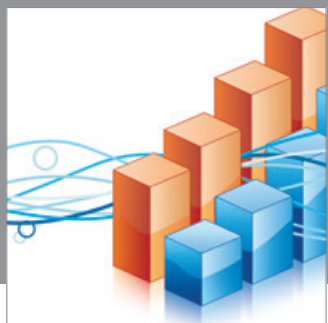

Advances in

Operations Research

mansans

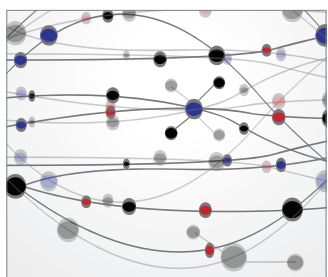

The Scientific World Journal
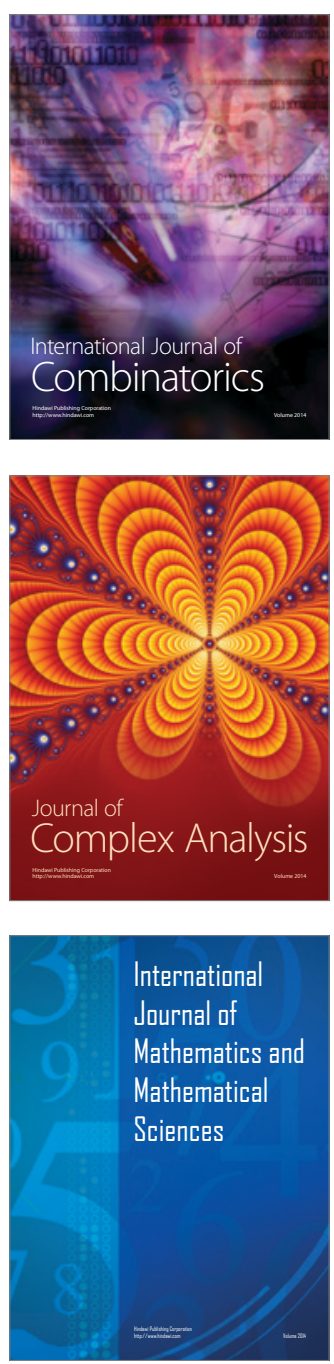
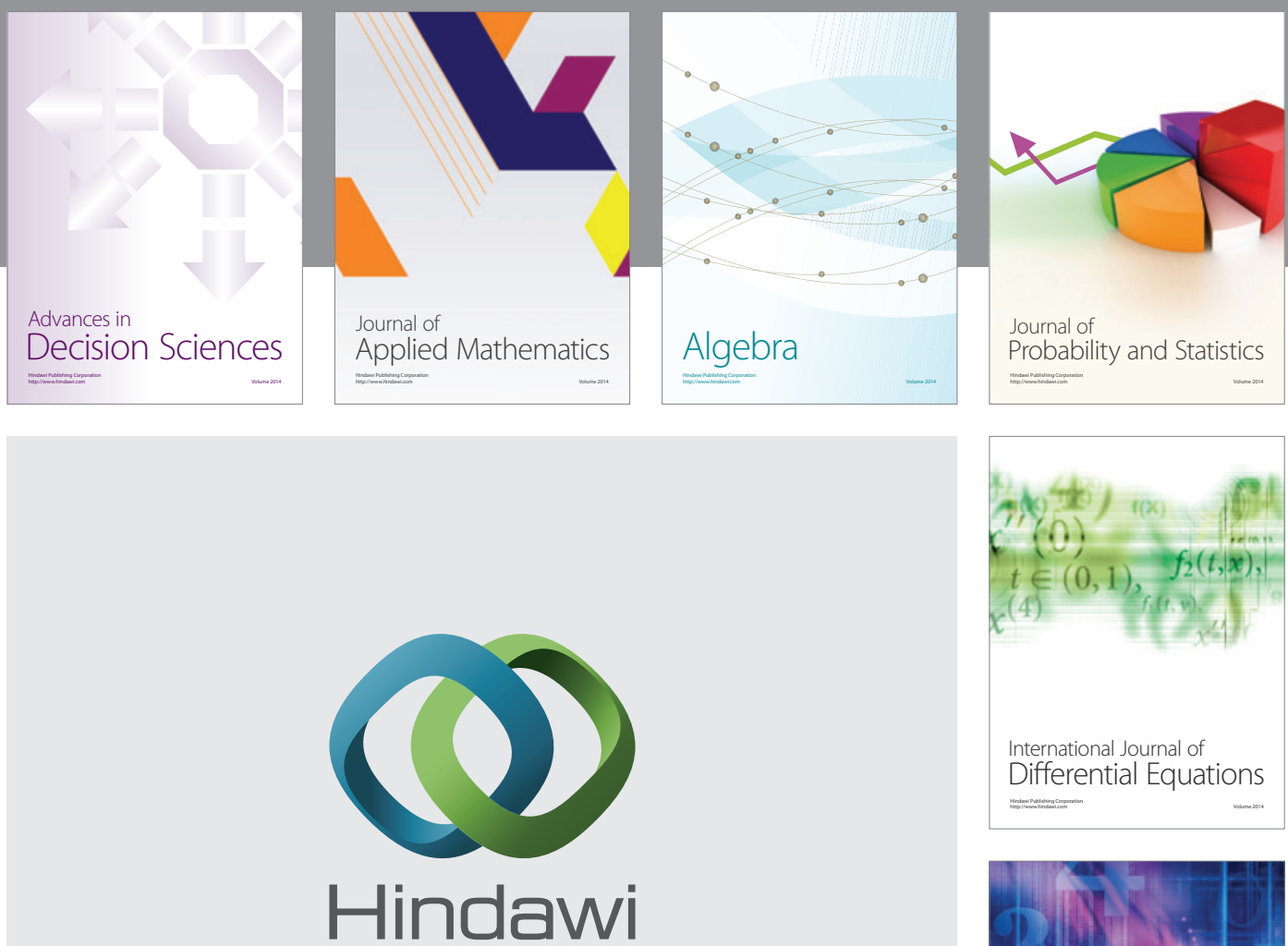

Submit your manuscripts at http://www.hindawi.com
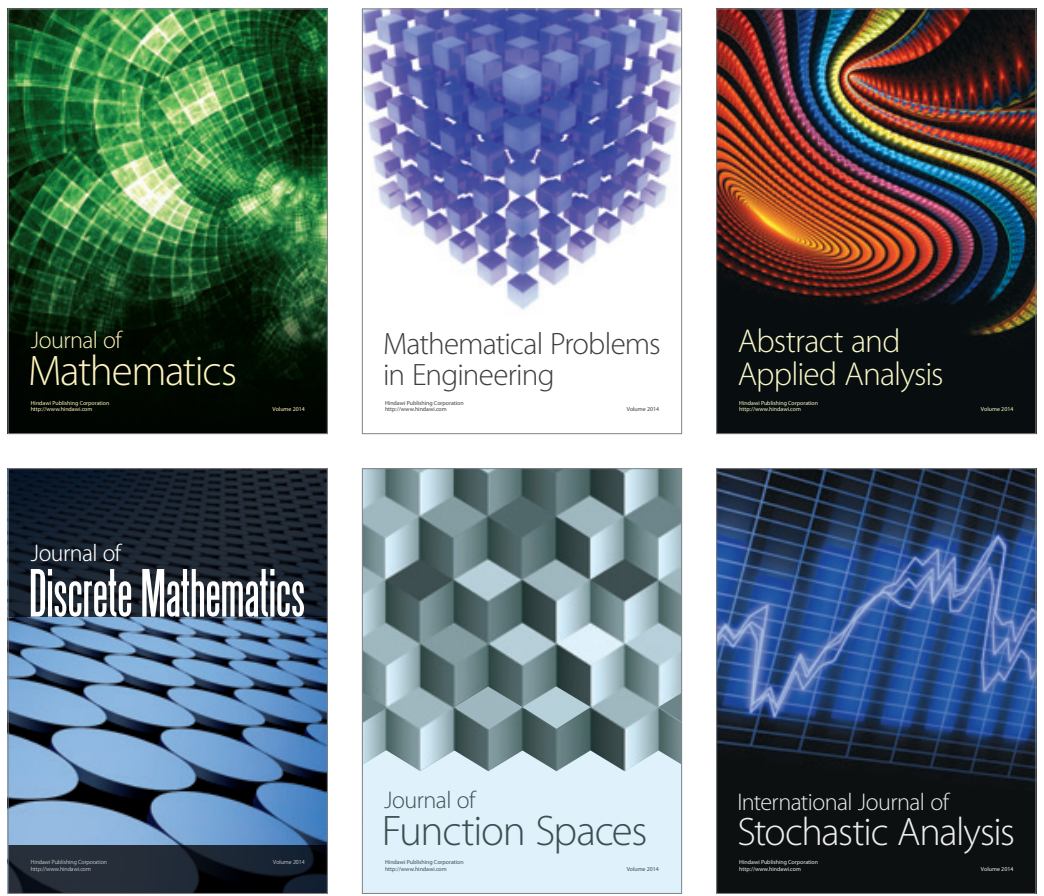

Journal of

Function Spaces

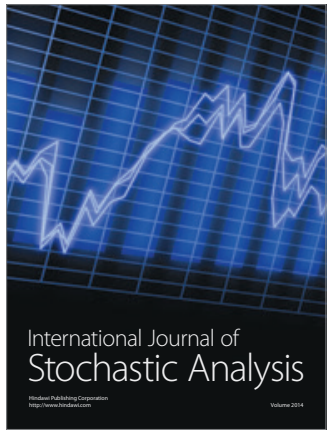

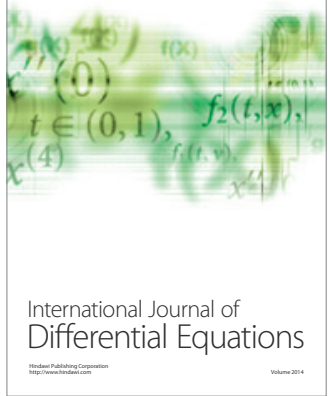
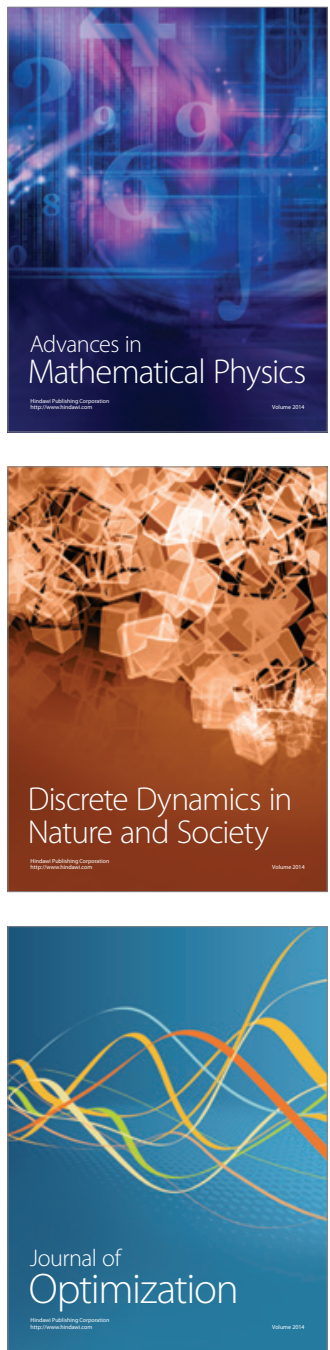